\title{
A High-Order Well-Balanced Discontinuous Galerkin Method Based on the Hydrostatic Reconstruction for the Ripa Model
}

\author{
Jiaojiao $\mathrm{Li}^{1}$, Gang $\mathrm{Li}^{1, *}$, Shouguo Qian ${ }^{1, *}$, Jinmei $\mathrm{Gao}^{1}$ \\ and Qiang $\mathrm{Niu}^{2}$ \\ ${ }^{1}$ School of Mathematics and Statistics, Qingdao University, Qingdao, \\ Shandong 266071, China \\ ${ }^{2}$ Department of Mathematical Sciences, Xi'an Jiaotong-Liverpool University, \\ Suzhou, Jiangsu 215123, China
}

Received 21 July 2019; Accepted (in revised version) 7 January 2020

\begin{abstract}
In this work, we present a high-order discontinuous Galerkin method for the shallow water equations incorporating horizontal temperature gradients (also known as the Ripa model), which exactly maintains the lake at rest steady state. Herein, we propose original numerical fluxes defined on the basis of the hydrostatic reconstruction idea and a simple source term approximation. This novel approach allows us to achieve the well-balancing of the discontinuous Galerkin method without complication. Moreover, the proposed method retains genuinely high-order accuracy for smooth solutions and it shows good resolution for discontinuous solutions at the same time. Rigorous numerical analysis as well as extensive numerical results all verify the good performances of the proposed method.
\end{abstract}

AMS subject classifications: 74S05

Key words: Ripa model, lake at rest steady state, source term, discontinuous Galerkin method, well-balancing property, hydrostatic reconstruction.

\section{Introduction}

Numerical simulation by high-order methods has a wide range of applications in environmental hydrodynamics [1,2]. Herein, we deal with the Ripa model, which is deduced from the shallow water equations (SWE) by incorporating the horizontal temperature gradients [3-5], often used to study ocean currents.

${ }^{*}$ Corresponding author. Emails: jiaojiaojiao807@163.com (J. J. Li), gangli1978@163.com (G. Li), sgqian1976@163.com (S. G. Qian), gjinmei1979@163.com (J. M. Gao), qiang.niu@xjtlu.edu.cn (Q. Niu) 
The one-dimensional Ripa model has the following form:

$$
\left\{\begin{array}{l}
h_{t}+(h u)_{x}=0 \\
(h u)_{t}+\left(h u^{2}+\frac{1}{2} g \theta h^{2}\right)_{x}=-g \theta h B_{x} \\
(h \theta)_{t}+(h u \theta)_{x}=0
\end{array}\right.
$$

where $x$ is the space, $t$ is the time, $h$ represents the water depth, $u$ is the flow velocity, $B$ is the bottom topography, and $g$ is the gravitational constant. Correspondingly, $h+$ $B$ stands for the free water surface level. Here, $\theta=\frac{\bar{\Theta}-\Theta}{\bar{\Theta}}$, with $\Theta$ and $\bar{\Theta}$ denoting the potential temperature and the reference potential temperature, accounts for the effects of the temperature on the fluid density. In addition, $P=\frac{1}{2} g \theta h^{2}$ represents the resultant of the pressure on each vertical divided by a reference fluid density. For the sake of easy presentation, we rewrite the system (1.1) in a compact vector form

$$
U_{t}+F(U)_{x}=S(B, U)
$$

with

$$
U=(h, h u, h \theta)^{\mathrm{T}}, \quad F(U)=\left(h u, h u^{2}+\frac{1}{2} g \theta h^{2}, h u \theta\right)^{\mathrm{T}} \quad \text { and } \quad S(B, U)=\left(0,-g \theta h B_{x}, 0\right)^{\mathrm{T}}
$$

being the vector of the conservative variables, the physical flux, as well as the vector of the source terms, respectively.

From the mathematical point of view, the system (1.1) combines hyperbolic balance laws, which are associated to particular steady states when $U_{t}=0$. Especially, the onedimensional Ripa model (1.1) admits the following two types of lake at rest steady states [6]:

$$
\begin{aligned}
& u=0, \quad \theta=\text { constant }, \quad h+B=\text { constant } \\
& u=0, \quad B=\text { constant }, \quad P=\frac{1}{2} g \theta h^{2}=\text { constant. }
\end{aligned}
$$

Under the steady state (1.3a), the flux gradient is non-zero and is exactly balanced by the source term (i.e., $F(U)_{x}=S(B, U)$ ).

To reproduce this asymptotic behavior of the system (1.1), the well-balancing property of a numerical method, which is the property to maintain the exact balance between the flux gradient and the source term at the discrete level accurately preserving the steady state up to the machine accuracy, is welcome. A numerical method satisfying the well-balancing property is named well-balanced method. Moreover, it is important to note that, compared with the non well-balanced counterparts, the well-balanced methods can accurately resolve small perturbations of the steady state on relatively coarse meshes [7-9], saving computational efforts considerably. 
The balance between the flux gradient and the source term involves terms that are not a polynomial functions and therefore can not be exactly represented in schemes involving polynomial reconstructions such as the the discontinuous Galerkin (DG) methods [10] or the weighted essentially non-oscillatory (WENO) schemes [11]. Therefore, the above feature brings difficulties to construct high-order methods. In fact, the coupling of traditional methods for the numerical flux computation with a straightforward source term treatment destroys the delicate balance in its discretized version, as highlighted by the apparition of spurious numerical oscillations. Even with the mesh refinement, the oscillations will only diminish, but will not disappear. In any case, the mesh refinement beyond some thresholds is impractical for two-dimensional problems due to the excessive computational cost.

In the contexts of general hyperbolic equations and especially in the SWE framework, to overcome the shortcoming of the traditional methods, specific well-balanced methods [12-15] have been originally presented.

In recent years, there have been some interesting attempts on the construction of well-balanced schemes also for the Ripa model. For example, Chertock et al. [16] present well-balanced central-upwind schemes with an interface tracking technique originally developed in [17]. Moreover, Touma and Klingenberg [18] develop secondorder well-balanced finite volume models under the framework of unstaggered central schemes. Sánchez-Linares et al. [19] develop a HLLC scheme based on the theory of path-conservative approximate Riemann solvers. Recently, Han and Li [20] design wellbalanced finite difference WENO schemes with the help of a special splitting of the source term. More recently, Qian et al. [21] propose well-balanced DG methods coupled with a special splitting of the source term.

The above schemes are either finite volume schemes or finite difference schemes. Among the high-order numerical methods in the literature, discontinuous Galerkin methods are a class of finite element methods using discontinuous piecewise polynomial space as the solution and test function spaces, which combine advantages of both finite element methods and finite volume schemes (see $[22,23]$ for a brief historic review). The DG methods possess the following advantages: high-order accuracy, easy parallel implementation, flexibility for hp-adaptation, convenient treatment for the boundary conditions on arbitrary geometry. In recent years, the above advantages make the DG methods popular in the fields of computational hydrodynamics [24-32].

The key objective of this research is to develop a high-order DG method for the Ripa model, well-balanced for the lake at rest steady state (1.3a). Compared with [21], the main novel aspect of this study is the definition of numerical fluxes based on the hydrostatic reconstruction idea [33]. The proposed numerical flux coupled with a plain treatment of the source term allows to satisfy the well-balancing property with an efficient and simple scheme. Furthermore, the resulting method preserves high-order accuracy in case of smooth solutions and a sharp reproduction of the solution discontinuities.

The structure of the paper is as follows. In Section 2, we present the well-balanced DG method for one-dimensional Ripa model and then extend it to the two-dimensional 
case. In Section 3, we carry out extensive numerical experiments to demonstrate the performances of the resulting DG method. Finally, brief conclusions are drawn in Section 4.

\section{Construction of well-balanced DG method for the Ripa model}

In this section, we present the well-balanced DG method for the one- and twodimensional Ripa model.

\subsection{Notations}

We start by presenting the standard notations under the framework of the DG methods. We first divide the interval $I=[a, b]$ into $N$ subintervals and denote the cells by $I_{j}=\left[x_{j-\frac{1}{2}}, x_{j+\frac{1}{2}}\right]$ for $j=1, \cdots, N$. The center of each cell is $x_{j}=\frac{1}{2}\left(x_{j-\frac{1}{2}}+x_{j+\frac{1}{2}}\right)$, and the mesh size is denoted by $\Delta x_{j}=x_{j+\frac{1}{2}}-x_{j-\frac{1}{2}}$ with $\Delta x=\max _{1 \leq j \leq N} \Delta x_{j}$ being the maximum mesh size. The piecewise polynomial space $V_{h}^{k}$ is defined as the space of polynomials with degree up to $k$ in each cell $I_{j}$, that is

$$
V_{h}^{k}=\left\{\phi:\left.\phi\right|_{I_{j}} \in P^{k}\left(I_{j}\right), j=1, \cdots, N\right\} .
$$

Note that the polynomials in $V_{h}^{k}$ are allowed to have discontinuities across element interfaces. In the context of the DG methods, the numerical approximation to any variable $U$ is denoted by $U_{h}$, which belongs to the finite element space $V_{h}^{k}$. In addition, we denote by $U_{h, j+\frac{1}{2}}^{+}$and $U_{h, j+\frac{1}{2}}^{-}$the values of $U_{h}$ at the cell interface $x_{j+\frac{1}{2}}$ from the right cell $I_{j+1}$ and from the left cell $I_{j}$, respectively. In this work, we consider a third-order method, i.e., $k=2$.

\subsection{Well-balanced DG method for the one-dimensional case}

The standard DG method [22] for the one-dimensional Ripa model (1.1) is given by

$$
\int_{I_{j}}\left(U_{h}\right)_{t} \phi d x-\int_{I_{j}} F\left(U_{h}\right) \phi_{x} d x+\widehat{F}_{j+\frac{1}{2}} \phi\left(x_{j+\frac{1}{2}}^{-}\right)-\widehat{F}_{j-\frac{1}{2}} \phi\left(x_{j-\frac{1}{2}}^{+}\right)=\int_{I_{j}} S\left(U_{h}, B_{h}\right) \phi d x,
$$

with $\phi(x) \in V_{h}^{k}$ being a test function, and $\widehat{F}_{j+\frac{1}{2}}=\hat{f}\left(U_{h, j+\frac{1}{2}}^{-}, U_{h, j+\frac{1}{2}}^{+}\right)$stands for the numerical flux. The numerical flux $\hat{f}\left(a_{1}, a_{2}\right)$ should be monotone, consistent and Lipschitz continuous. In this study, we apply the Lax-Friedrichs flux

$$
\hat{f}\left(a_{1}, a_{2}\right)=\frac{1}{2}\left(F\left(a_{1}\right)+F\left(a_{2}\right)-\alpha\left(a_{2}-a_{1}\right)\right),
$$


where $\alpha=\max _{x}|\lambda(U)|$ with $\lambda(U)$ being the eigenvalues of the Jacobian $F^{\prime}(U)$, and the maximum is taken over the whole computational domain. The eigenvalues of the Jacobian matrices subject to the one- and two-dimensional Ripa models are given in Appendix $\mathrm{A}$ and $\mathrm{B}$.

However, the above standard DG method fails to maintain the well-balancing property. Herein, inspired by the hydrostatic reconstruction idea in [33], our semi-discrete DG method for the one-dimensional Ripa model (1.1) is defined as follows: for any test function $\phi(x) \in V_{h}^{k}, U_{h}$ is given by

$$
\int_{I_{j}}\left(U_{h}\right)_{t} \phi d x-\int_{I_{j}} F\left(U_{h}\right) \phi_{x} d x+\widehat{F}_{j+\frac{1}{2}}^{l} \phi\left(x_{j+\frac{1}{2}}^{-}\right)-\widehat{F}_{j-\frac{1}{2}}^{r} \phi\left(x_{j-\frac{1}{2}}^{+}\right)=\int_{I_{j}} S\left(U_{h}, B_{h}\right) \phi d x,
$$

where $\widehat{F}_{j+\frac{1}{2}}^{l}$ and $\widehat{F}_{j-\frac{1}{2}}^{r}$ are numerical fluxes defined in (2.6).

\subsubsection{Well-balanced numerical fluxes via hydrostatic reconstruction}

The form of $\widehat{F}_{j-\frac{1}{2}}^{r}$ and $\widehat{F}_{j+\frac{1}{2}}^{l}$ in (2.4) is the key element to achieve the well-balancing property. As a matter of fact, our key idea is to make sure that for the lake at rest steady state (1.3a) the following equalities:

$$
\widehat{F}_{j+\frac{1}{2}}^{l}=F\left(U_{h, j+\frac{1}{2}}^{-}\right) \quad \text { and } \quad \widehat{F}_{j-\frac{1}{2}}^{r}=F\left(U_{h, j-\frac{1}{2}}^{+}\right),
$$

are true. Subsequently, we will take some procedures to realize the objective (2.5).

To achieve this aim, we write the numerical fluxes in (2.4) as:

$$
\begin{aligned}
& \widehat{F}_{j+\frac{1}{2}}^{l}=\hat{f}\left(U_{j+\frac{1}{2}}^{*,-}, U_{j+\frac{1}{2}}^{*,+}\right)+F\left(U_{h, j+\frac{1}{2}}^{-}\right)-F\left(U_{j+\frac{1}{2}}^{*,-}\right), \\
& \widehat{F}_{j-\frac{1}{2}}^{r}=\hat{f}\left(U_{j-\frac{1}{2}}^{*,-}, U_{j-\frac{1}{2}}^{*,+}\right)+F\left(U_{h, j-\frac{1}{2}}^{+}\right)-F\left(U_{j-\frac{1}{2}}^{*,+}\right) .
\end{aligned}
$$

At each time level, the cell interface values $U_{h, j+\frac{1}{2}}^{ \pm}$can be computed from the piecewise polynomial solution $U_{h}$ directly. In order to maintain the well-balancing property, we redefine locally updated cell interface values based on the hydrostatic reconstruction idea [33]. Therefore, we propose to define:

$$
\begin{aligned}
& h_{j+\frac{1}{2}}^{*,+}=\max \left(0, h_{h, j+\frac{1}{2}}^{+}+B_{h, j+\frac{1}{2}}^{+}-\max \left(B_{h, j+\frac{1}{2}}^{-}, B_{h, j+\frac{1}{2}}^{+}\right)\right), \\
& h_{j+\frac{1}{2}}^{*,-}=\max \left(0, h_{h, j+\frac{1}{2}}^{-}+B_{h, j+\frac{1}{2}}^{-}-\max \left(B_{h, j+\frac{1}{2}}^{-}, B_{h, j+\frac{1}{2}}^{+}\right)\right),
\end{aligned}
$$

as well as

$$
\theta_{j+\frac{1}{2}}^{*}=\max \left(\theta_{h, j+\frac{1}{2}}^{-}, \theta_{h, j+\frac{1}{2}}^{+}\right)
$$


Then, we redefine the left and right values of $U_{h}$ at the cell interface as follows:

$$
U_{j+\frac{1}{2}}^{*, \pm}=\left(\begin{array}{c}
h_{j+\frac{1}{2}}^{*, \pm} \\
(h u)_{h, j+\frac{1}{2}}^{ \pm} \\
h_{j+\frac{1}{2}}^{* \pm} \theta_{j+\frac{1}{2}}^{*}
\end{array}\right) \quad \text { and } \quad U_{j-\frac{1}{2}}^{*, \pm}=\left(\begin{array}{c}
h_{j-\frac{1}{2}}^{*, \pm} \\
(h u)_{h, j-\frac{1}{2}}^{ \pm} \\
h_{j-\frac{1}{2}}^{*, \pm} \theta_{j-\frac{1}{2}}^{*}
\end{array}\right) .
$$

The integral of the source term in (2.4) is approximated by a straightforward third-order accurate Gaussian quadrature.

\subsubsection{Temporal discretization and slope limiter}

The semi-discrete DG method is given by (2.4), equipped with the numerical fluxes $\widehat{F}^{l}$ and $\widehat{F}^{r}$ computed in (2.6) as well as a simple numerical approximation of the source term. The final DG method is completed by integrating in time the semi-discrete model with a high-order total variation diminishing (TVD) Runge-Kutta method [34]. In all the computations, we apply the following third-order Runge-Kutta temporal discretization:

$$
\begin{aligned}
& U^{(1)}=U^{n}+\Delta t \mathcal{F}\left(U^{n}\right), \\
& U^{(2)}=\frac{3}{4} U^{n}+\frac{1}{4}\left(U^{(1)}+\Delta t \mathcal{F}\left(U^{(1)}\right)\right), \\
& U^{n+1}=\frac{1}{3} U^{n}+\frac{2}{3}\left(U^{(2)}+\Delta t \mathcal{F}\left(U^{(2)}\right)\right),
\end{aligned}
$$

with $\mathcal{F}(U)$ being the semi-discrete DG model.

Moreover, in the context of DG methods, a slope limiter is indispensable in the case of discontinuous solutions and is usually performed after each Runge-Kutta stage on the solution $U_{h}$. However, the standard limiter might destroy the preservation of the steady state (1.3a). Therefore, following the idea presented in [33], we implement the limiter procedure on the function $\left(h_{h}+B_{h},(h u)_{h},(h \theta)_{h}+(B \theta)_{h}\right)^{T}$ instead. The modified DG solutions are then defined by

$$
\begin{aligned}
& h_{h}^{\text {mod }}=(h+B)_{h}^{\bmod }-B_{h}, \\
& (h \theta)_{h}^{\bmod }=\left((h \theta)_{h}+(B \theta)_{h}\right)^{\text {mod }}-(B \theta)_{h} .
\end{aligned}
$$

Because

$$
\begin{aligned}
& {\overline{h_{h}}}^{\text {mod }}=\overline{(h+B)_{h}}{ }^{\text {mod }}-\overline{B_{h}}=\overline{(h+B)_{h}}-\overline{B_{h}}=\overline{h_{h}}, \\
& {\overline{(h \theta)_{h}}}^{\text {mod }}=\overline{(h \theta)_{h}+(B \theta)_{h}}{ }^{\text {mod }}-\overline{(B \theta)_{h}}=\overline{(h \theta)_{h}+(B \theta)_{h}}-\overline{(B \theta)_{h}}=\overline{(h \theta)_{h}},
\end{aligned}
$$

due to $h+B=$ constant and $\theta=$ constant in (1.3a), we observe that the current steps will not destroy the conservativity of $h_{h}$ and $(h \theta)_{h}$, which should be maintained during the limiter procedures. In this article, we employ the total variation bounded (TVB) limiter presented in $[35,36]$. 


\subsubsection{Analytical analysis of the well-balancing property}

This implementation leads to a well-balanced DG method for the one-dimensional Ripa model, as outlined in the following proposition.

Proposition 2.1. For the one-dimensional Ripa model (1.1), the semi-discrete DG method (2.4) in combination with the numerical fluxes (2.6) based on the hydrostatic reconstruction idea as well as a simple source term approximation, maintains the well-balancing property for the lake at rest steady state (1.3a).

Proof. For the lake at rest steady state (1.3a), the first equation $(h u)_{x}=0$ and the third equation $(h u \theta)_{x}=0$ are satisfied exactly for any consistent numerical flux, since $h u=0$ and $h u \theta=0$ due to the imposed quiescent flow $(u=0)$. Therefore, only the second equation requires particular attention.

At the steady state (1.3a), we first have $u_{h}=0$, which leads to

$$
(h u)_{h, j+\frac{1}{2}}^{-}=(h u)_{h, j+\frac{1}{2}}^{+}=0 .
$$

In addition, we also have

$$
h_{h, j+\frac{1}{2}}^{-}+B_{h, j+\frac{1}{2}}^{-}=h_{h, j+\frac{1}{2}}^{+}+B_{h, j+\frac{1}{2}}^{+}=\text {constant. }
$$

Then, in combination with (2.8), we can get

$$
h_{j+\frac{1}{2}}^{*,-}=h_{j+\frac{1}{2}}^{*,+}
$$

according to (2.7). In summary, we easily observe that

$$
U_{j+\frac{1}{2}}^{*,-}=U_{j+\frac{1}{2}}^{*,+}
$$

thanks to (2.9).

Subsequently, by means of the consistence of the Lax-Friedrichs flux (2.3), we can obtain

$$
\begin{aligned}
\widehat{F}_{j+\frac{1}{2}}^{l} & =\left(\begin{array}{c}
0 \\
\frac{1}{2} g\left(h_{j+\frac{1}{2}}^{*}\right)^{2} \theta_{j+\frac{1}{2}}^{*} \\
0
\end{array}\right)+\left(\begin{array}{c}
0 \\
\frac{1}{2} g\left(h_{j+\frac{1}{2}}^{-}\right)^{2} \theta_{j+\frac{1}{2}}^{-} \\
0
\end{array}\right)-\left(\begin{array}{c}
0 \\
\frac{1}{2} g\left(h_{j+\frac{1}{2}}^{*}\right)^{2} \theta_{j+\frac{1}{2}}^{*} \\
0
\end{array}\right) \\
& =\left(\begin{array}{c}
0 \\
\frac{1}{2} g\left(h_{j+\frac{1}{2}}^{-}\right)^{2} \theta_{j+\frac{1}{2}}^{-} \\
0
\end{array}\right)=F\left(U_{\left.h, j+\frac{1}{2}\right),}^{-}\right.
\end{aligned}
$$

due to (2.6). Similarly, we have

$$
\widehat{F}_{j-\frac{1}{2}}^{r}=F\left(U_{h, j-\frac{1}{2}}^{+}\right) .
$$


Furthermore, we here assume that $U_{h}$ is a steady state solution of the equation $U_{t}+$ $F\left(U_{h}\right)_{x}=S\left(B_{h}, U_{h}\right)$. In fact, this observation is true since $\theta_{h}=$ constant, $h_{h}+B_{h}=$ constant and $u_{h}=0$, which imply $\left(\frac{1}{2} g h_{h}^{2} \theta_{h}\right)_{x}=-g h_{h} \theta_{h}\left(B_{h}\right)_{x}$ in the second equation, or

$$
F\left(U_{h}\right)_{x}=S\left(B_{h}, U_{h}\right)
$$

for the whole system.

Subsequently, under the steady state and the above assumption, the residue $R$ between the approximation to both the flux gradient and the source term reduces to

$$
\begin{aligned}
R & =\widehat{F}_{j+\frac{1}{2}}^{l} \phi\left(x_{j+\frac{1}{2}}^{-}\right)-\widehat{F}_{j-\frac{1}{2}}^{r} \phi\left(x_{j-\frac{1}{2}}^{+}\right)-\int_{I_{j}} F\left(U_{h}\right) \phi_{x} d x-\int_{I_{j}} S\left(B_{h}, U_{h}\right) \phi d x \\
& =F\left(U_{h, j+\frac{1}{2}}^{-}\right) \phi\left(x_{j+\frac{1}{2}}^{-}\right)-F\left(U_{h, j-\frac{1}{2}}^{+}\right) \phi\left(x_{j-\frac{1}{2}}^{+}\right)-\int_{I_{j}} F\left(U_{h}\right) \phi_{x} d x-\int_{I_{j}} S\left(B_{h}, U_{h}\right) \phi d x \\
& =\int_{I_{j}} F\left(U_{h}\right)_{x} \phi d x-\int_{I_{j}} S\left(B_{h}, U_{h}\right) \phi d x \\
& =\int_{I_{j}}\left(F\left(U_{h}\right)_{x}-S\left(B_{h}, U_{h}\right)\right) \phi d x=0,
\end{aligned}
$$

where the second equality is due to (2.11) and (2.12), the third equality follows from a simple integration by parts, and the last equality derives from the equality (2.13).

\subsubsection{Summary of the DG method}

Finally, we summarize the complete procedure to obtain the proposed DG method for the one-dimensional Ripa model:

1. Compute the $L^{2}$ projection of the initial data $U(x, 0)$ and of the bottom topography $B(x)$, and denote them by $U_{h}$ and $B_{h}$, respectively;

2. Compute the well-balanced numerical fluxes (2.6) following the hydrostatic reconstruction idea;

3. Evaluate the source term approximation by a third-order accuracy Gaussian quadrature;

4. Apply the third-order TVD Runge-Kutta method (2.10) to advance the solution in time;

5. Apply the slope limiter procedure, and repeat steps $2-5$. 


\subsection{Extension to the two-dimensional case}

The two-dimensional Ripa model has the following form:

$$
\left\{\begin{array}{l}
h_{t}+(h u)_{x}+(h v)_{y}=0 \\
(h u)_{t}+\left(h u^{2}+\frac{1}{2} g \theta h^{2}\right)_{x}+(h u v)_{y}=-g \theta h B_{x} \\
(h v)_{t}+(h u v)_{x}+\left(h v^{2}+\frac{1}{2} g \theta h^{2}\right)_{y}=-g \theta h B_{y} \\
(h \theta)_{t}+(h u \theta)_{x}+(h v \theta)_{y}=0
\end{array}\right.
$$

where $v$ stands for the flow velocity in the $y$-direction, and the remaining notations are the same as in the one-dimensional model (1.1). The two-dimensional Ripa model (2.14) admits the following lake at rest steady state:

$$
u=v=0, \quad \theta=\text { constant }, \quad h+B=\text { constant. }
$$

The extension of the proposed one-dimensional DG method to the two-dimensional case is straightforward. Furthermore, in a similar way, we can also easily prove that the wellbalancing property is still valid for the two-dimensional case.

\section{Numerical results}

In this Section, we implement extensive one- and two-dimensional numerical examples to validate the proposed DG method. To ensure the numerical stability, we adopt an adaptive time step $\Delta t^{n}$ to satisfy the following requirement

$$
\frac{\Delta t^{n}}{\Delta x} \max _{1 \leq j \leq N}\left(\left|\bar{u}_{j}^{n}\right|+\sqrt{g \bar{h}_{j}^{n}}\right)=\mathrm{CFL} .
$$

In all of the computations, we take the gravitational constant and the CFL number as $1 \mathrm{~m} / \mathrm{s}^{2}$ and 0.18 , respectively.

\subsection{One-dimensional cases}

We first present some numerical results for the one-dimensional Ripa model (1.1).

\subsubsection{Testing the well-balancing property}

The main goal of this example is to experimentally verify the well-balancing of the resulting DG method. Herein, we consider two bottom topographies, a smooth bottom

$$
B(x)=5 e^{-\frac{2}{5}(x-5)^{2}} \mathrm{~m}
$$


Table 1: $L^{1}$ and $L^{\infty}$ errors for different precisions for the lake at rest steady solutions over the smooth bottom topography (3.1) in Section 3.1.1.

\begin{tabular}{||c|ccc|ccc||}
\hline \multirow{2}{*}{ Precision } & \multicolumn{3}{|c|}{$L^{1}$ errors } & \multicolumn{3}{c||}{$L^{\infty}$ errors } \\
\cline { 2 - 7 } & $h$ & $h u$ & $h \theta$ & $h$ & $h u$ & $h \theta$ \\
\hline Single & $3.42 \times 10^{-7}$ & $3.67 \times 10^{-7}$ & $3.39 \times 10^{-7}$ & $1.31 \times 10^{-7}$ & $4.12 \times 10^{-7}$ & $2.21 \times 10^{-7}$ \\
\hline Double & $7.35 \times 10^{-15}$ & $6.21 \times 10^{-15}$ & $8.13 \times 10^{-15}$ & $2.81 \times 10^{-15}$ & $5.51 \times 10^{-15}$ & $3.24 \times 10^{-15}$ \\
\hline
\end{tabular}

Table 2: $L^{1}$ and $L^{\infty}$ errors for different precisions for the lake at rest steady solutions over the discontinuous bottom topography (3.2) in Section 3.1.1.

\begin{tabular}{||c|ccc|ccc||}
\hline \multirow{2}{*}{ Precision } & \multicolumn{3}{|c|}{$L^{1}$ errors } & \multicolumn{3}{c||}{$L^{\infty}$ errors } \\
\cline { 2 - 7 } & $h$ & $h u$ & $h \theta$ & $h$ & $h u$ & $h \theta$ \\
\hline Single & $2.22 \times 10^{-7}$ & $1.32 \times 10^{-7}$ & $2.85 \times 10^{-7}$ & $5.71 \times 10^{-7}$ & $3.35 \times 10^{-7}$ & $3.42 \times 10^{-7}$ \\
\hline Double & $3.11 \times 10^{-15}$ & $2.16 \times 10^{-15}$ & $3.43 \times 10^{-15}$ & $3.13 \times 10^{-15}$ & $6.18 \times 10^{-15}$ & $5.25 \times 10^{-15}$ \\
\hline
\end{tabular}

and a discontinuous one

$$
B(x)= \begin{cases}4 \mathrm{~m}, & \text { if } 4 \mathrm{~m} \leq x \leq 8 \mathrm{~m}, \\ 0 \mathrm{~m}, & \text { otherwise }\end{cases}
$$

The initial conditions are defined by

$$
h+B=10 \mathrm{~m}, \quad u=0 \mathrm{~m} / \mathrm{s} \text { and } \theta=0.1 .
$$

We consider the solution at $t=0.5 \mathrm{~s}$ on a computational domain $[0,10] \mathrm{m}$ discretized with 200 uniform cells. To show that the well-balancing property is maintained at the round-off error level, we apply single and double precisions to carry out the computation. We present the $L^{1}$ and $L^{\infty}$ errors for $h, h u$ and $h \theta$ in Tables 1 and 2 for the above two bottom topographies. We can obviously observe that the $L^{1}$ and $L^{\infty}$ errors are all at the level of round-off error even for different precisions, which verify that the resulting method satisfies the well-balancing property.

\subsubsection{Testing the accuracy order}

Here, we verify the third-order accuracy of the proposed method for a smooth solution. We take into account the following bottom topography and initial conditions:

$$
\begin{aligned}
& B(x)=\sin ^{2}(\pi x) \mathrm{m}, \\
& h(x, 0)=(5+\exp (\cos (2 \pi x))) \mathrm{m}, \\
& (h u)(x, 0)=\sin (\cos (2 \pi x)) \mathrm{m}^{2} / \mathrm{s}, \\
& \theta(x, 0)=\cos (2 \pi x),
\end{aligned}
$$

on an unitary computational domain $[0,1] \mathrm{m}$ discretized with an increasingly large number of cells (between 25 and 3200). We carry out the simulation until $t=0.1$ s with periodic 
Table 3: $L^{1}$ errors and accuracy orders for the test case in Section 3.1.2.

\begin{tabular}{||c|cc|cc|cc||}
\hline \multirow{2}{*}{$N$} & \multicolumn{2}{|c}{$h$} & \multicolumn{2}{c}{$h u$} & \multicolumn{2}{c||}{$h \theta$} \\
\cline { 2 - 8 } & $L^{1}$ error & Order & $L^{1}$ error & Order & $L^{1}$ error & Order \\
\hline 25 & $1.0005 \times 10^{-4}$ & & $7.9812 \times 10^{-5}$ & & $1.2137 \times 10^{-4}$ & \\
\hline 50 & $1.2512 \times 10^{-5}$ & 3.00 & $9.9459 \times 10^{-6}$ & 3.00 & $1.4590 \times 10^{-5}$ & 3.06 \\
\hline 100 & $1.5680 \times 10^{-6}$ & 3.00 & $1.2446 \times 10^{-6}$ & 3.00 & $1.7955 \times 10^{-6}$ & 3.02 \\
\hline 200 & $1.9678 \times 10^{-7}$ & 2.99 & $1.5614 \times 10^{-7}$ & 2.99 & $2.2412 \times 10^{-7}$ & 3.00 \\
\hline 400 & $2.4747 \times 10^{-8}$ & 2.99 & $1.9643 \times 10^{-8}$ & 2.99 & $2.8335 \times 10^{-8}$ & 2.98 \\
\hline 800 & $3.1177 \times 10^{-9}$ & 2.99 & $2.4767 \times 10^{-9}$ & 2.99 & $3.6133 \times 10^{-9}$ & 2.97 \\
\hline 1600 & $3.8970 \times 10^{-10}$ & 3.00 & $3.0970 \times 10^{-10}$ & 3.00 & $4.5415 \times 10^{-10}$ & 2.99 \\
\hline 3200 & $4.7743 \times 10^{-11}$ & 3.03 & $3.8041 \times 10^{-11}$ & 3.03 & $5.7659 \times 10^{-11}$ & 2.98 \\
\hline
\end{tabular}

boundary conditions. To obtain a reference solution, we apply the same method on a finer mesh with 6400 cells. In Table 3 , we show the $L^{1}$ errors and the accuracy order computed for $h, h u$ and $h \theta$. It is obvious that we get the expected third-order accuracy for this test case.

\subsubsection{Riemann problem over a flat bottom topography}

To verify the capability of the presented DG method to correctly reproduce rarefaction waves, shock waves and contact discontinuity waves, we consider a dam break problem over a flat bottom topography $(B=$ constant). The initial conditions are given as follows

$$
(h, u, \theta)(x, 0)= \begin{cases}(5 \mathrm{~m}, 0 \mathrm{~m} / \mathrm{s}, 3), & \text { if } x \leq 0 \mathrm{~m}, \\ (1 \mathrm{~m}, 0 \mathrm{~m} / \mathrm{s}, 5), & \text { otherwise }\end{cases}
$$

on the computational domain $[-1,1] \mathrm{m}$ discretized with 200 cells.

In Fig. 1, we compare the numerical results at $t=0.2 \mathrm{~s}$ with the exact solution obtained in [37]. For the sake of completeness, we also use as reference the results by the fifth-order well-balanced finite difference WENO scheme described in [20]. It is obvious that the results by the well-balanced DG method are in good agreement with both the analytical and numerical reference solutions. Moreover, it is important to note that the numerical results are resolved accurately and free of spurious numerical oscillations near the discontinuities, and are perfectly matched with other solutions by literature, e.g., [16].

\subsubsection{Dam break over a rectangular bump}

Here, we extend the dam break problem to the discontinuous bed case presented in [7]. The bottom topography is characterized by a rectangular bump:

$$
B(x)= \begin{cases}8 \mathrm{~m}, & \text { if }|x-300|<75 \mathrm{~m}, \\ 0 \mathrm{~m}, & \text { otherwise. }\end{cases}
$$



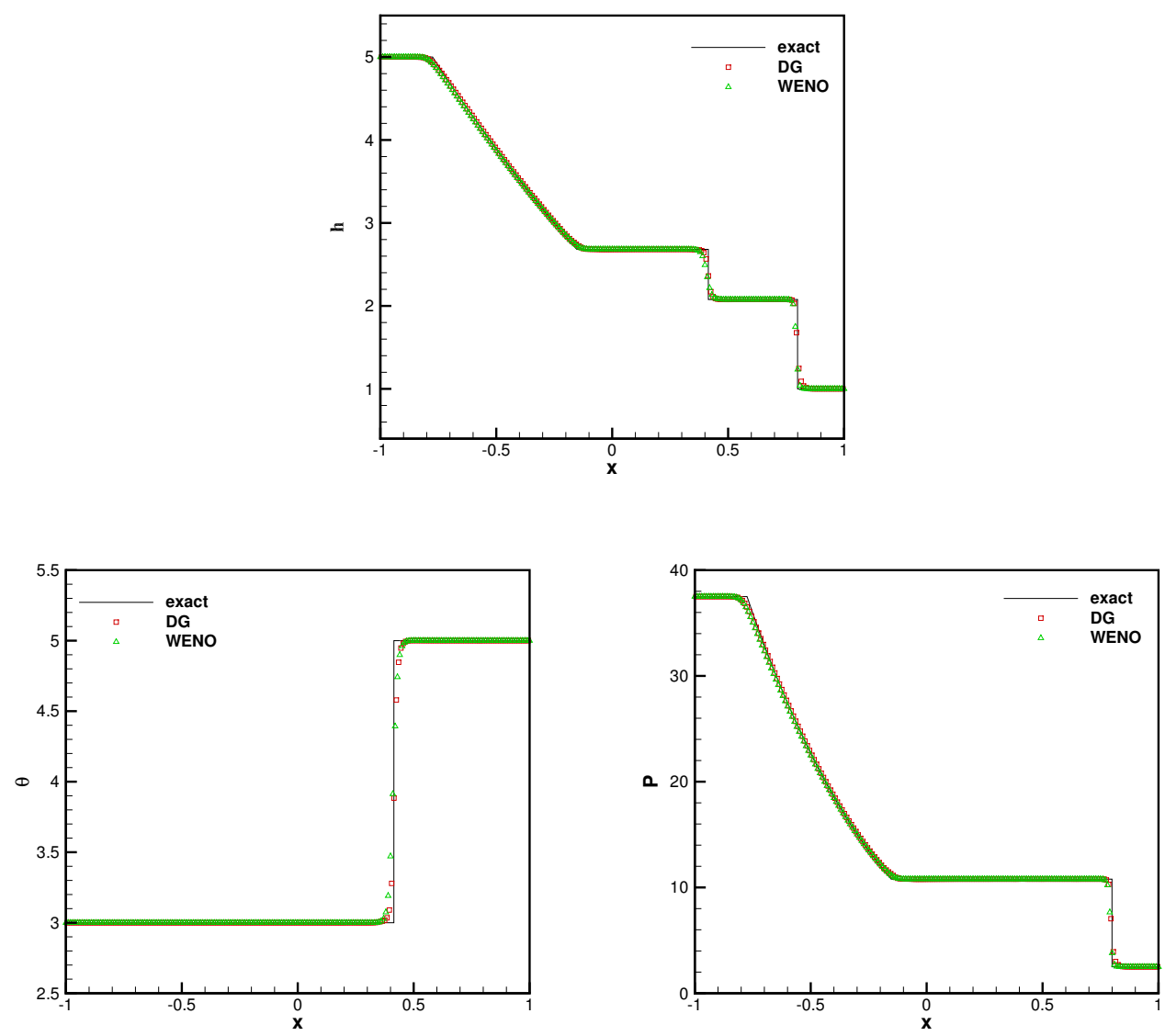

Figure 1: The numerical solution of Riemann problem over a flat bottom, Section 3.1.3, at $t=0.2 \mathrm{~s}$. $h$ (upper), $\theta$ (lower left) and $P=\frac{1}{2} g \theta h^{2}$ (lower right).

The initial conditions are defined as follows

$$
(h, u, \theta)= \begin{cases}((20-B(x)) \mathrm{m}, 0 \mathrm{~m} / \mathrm{s}, 10), & \text { if } x \leq 300 \mathrm{~m}, \\ ((15-B(x)) \mathrm{m}, 0 \mathrm{~m} / \mathrm{s}, 5), & \text { otherwise }\end{cases}
$$

on a computational domain $[0,600] \mathrm{m}$. In Fig. 2, we show the numerical results at $t=0.2 \mathrm{~s}$ using 400 cells against the reference solution obtained on a mesh with 2000 cells. For comparison, we also present the numerical solutions by the fifth-order well-balanced finite difference WENO schemes described in [20] on a mesh with 400 cells. We can clearly observe that the current method correctly reproduces sharp discontinuity in the solution due to the bed discontinuity in terms of water depth. 

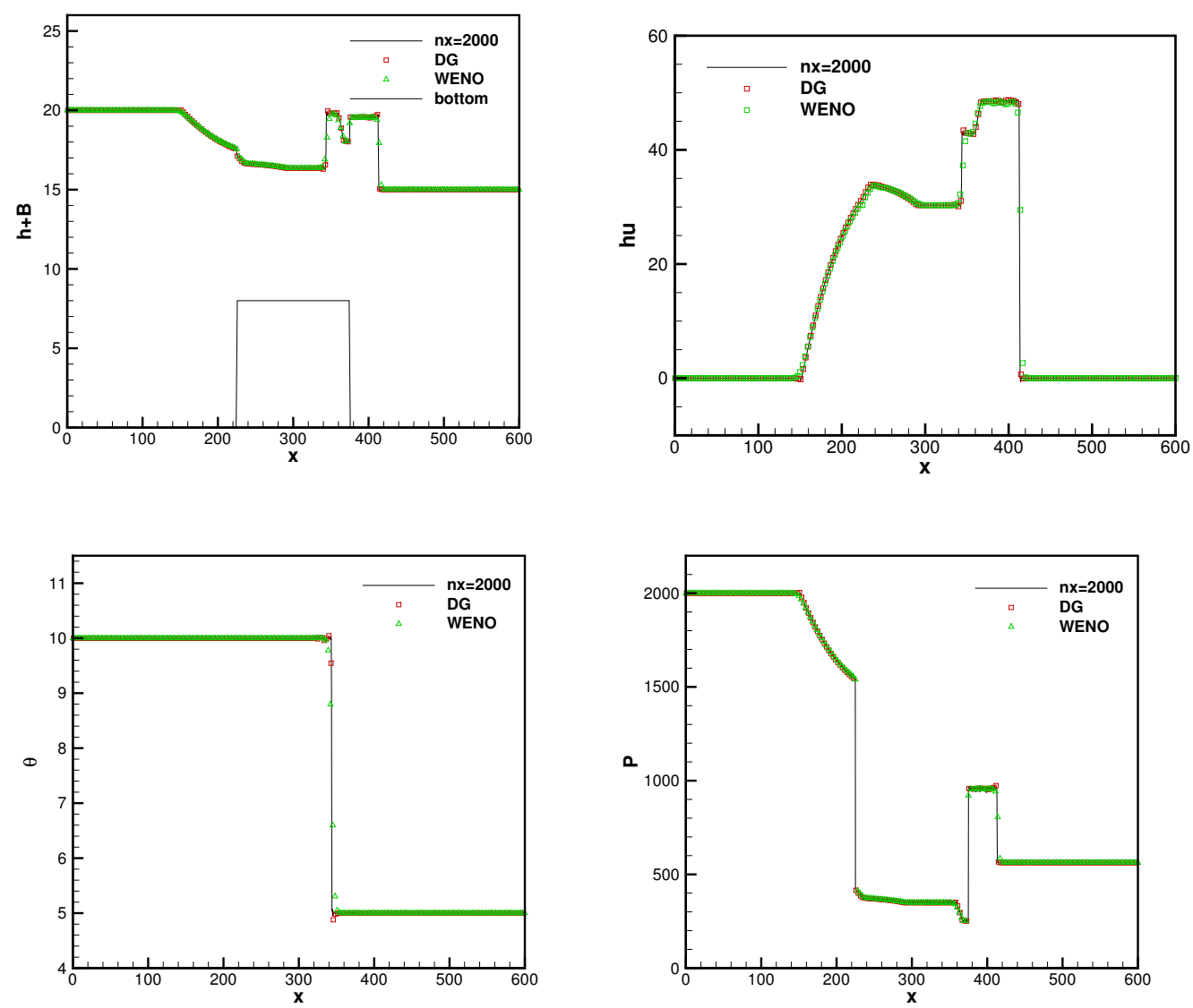

Figure 2: The numerical results of dam break problem over a rectangular bump, Section 3.1.4, at $t=0.2 \mathrm{~s}$. $h+B$ (upper left), $h u$ (upper right), $\theta$ (lower left) and $P=\frac{1}{2} g \theta h^{2}$ (lower right).

\subsubsection{Perturbation of a steady problem}

This experiment consists of a small perturbation to a steady problem as considered in [6, $16,19]$. The non-flat bottom topography is given by

$$
B(x)= \begin{cases}0.85\{\cos [10 \pi(x+0.9)]+1\} \mathrm{m}, & \text { if }-1 \mathrm{~m} \leq x \leq-0.8 \mathrm{~m}, \\ 1.25\{\cos [10 \pi(x-0.4)]+1\} \mathrm{m}, & \text { if } 0.3 \mathrm{~m} \leq x \leq 0.5 \mathrm{~m}, \\ 0 \mathrm{~m}, & \text { otherwise }\end{cases}
$$

It is clear that

$$
\left(h_{s}, u_{s}, \theta_{s}\right)(x)= \begin{cases}((6-B(x)) \mathrm{m}, 0 \mathrm{~m} / \mathrm{s}, 4), & \text { if } x<0 \mathrm{~m}, \\ ((4-B(x)) \mathrm{m}, 0 \mathrm{~m} / \mathrm{s}, 9), & \text { otherwise }\end{cases}
$$



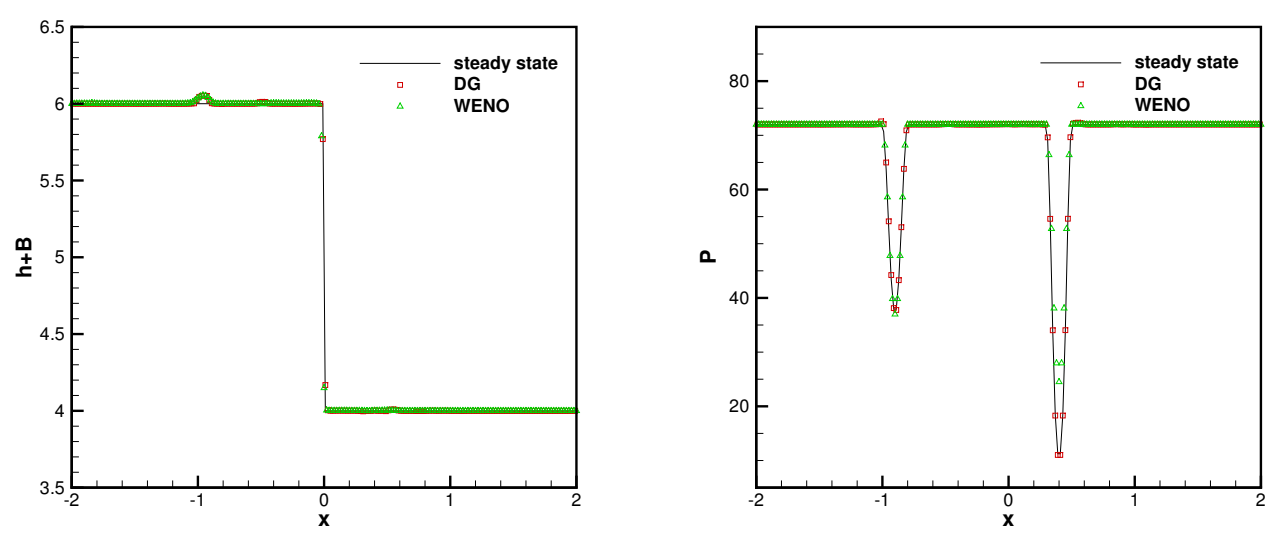

Figure 3: Results of the small perturbation of a steady state solution in Section 3.1 .5 at time $t=0.1$ s. $h+B$ (left) and $P=\frac{1}{2} g \theta h^{2}$ (right).

is a non-moving piecewise constant steady state solution. In fact, this solution is obtained by combining two lake at rest states of the type of (1.3a) joined through a discontinuity that satisfies (1.3b). We perturb this steady state imposing the following initial data

$$
\left.(h, u, \theta)^{\top}(x, 0)=\left(h_{s}, u_{s}, \theta_{s}\right)^{\top}(x)+(0.1 \mathrm{~m}, 0 \mathrm{~m} / \mathrm{s}, 0)\right)^{\top} \chi_{[-1.5,-1.4]}(x),
$$

with $\chi_{[-1.5,-1.4]}(x)=1$ if $x \in[-1.5,-1.4] \mathrm{m}$ and $\chi_{[-1.5,-1.4]}(x)=0$ elsewhere on a computational domain. We perform a simulation on the computational domain $[-2,2] \mathrm{m}$ discretized with 200 cells. We show the results at $t=0.1 \mathrm{~s}$ in Fig. 3 . It is evident that, due to the well-balancing property, the method can reproduce the propagation of small perturbations of the steady state (1.3a), for $x<0$ and $x>0$, also on non-flat bottoms.

Interestingly, this test shows also that the proposed DG method captures the stationary contact discontinuity of type (1.3b), at $x=0$, with negligible numerical diffusivity, showing an essentially non-oscillatory property. In this case the convergence to the nonmoving solution is not exact at the discrete level but remains very good.

In the same Fig. 3, for completeness, we also report the results by the fifth-order wellbalanced finite difference WENO scheme [20] on a mesh with 200 cells.

\subsection{Two-dimensional cases}

In this section, we consider the two-dimensional Ripa model. 


\subsubsection{Testing the well-balancing property}

We consider a steady state problem over a bottom topography that consists of two Gaussian shaped humps

$$
B(x, y)= \begin{cases}0.5 \exp \left(-100\left((x+0.5)^{2}+(y+0.5)^{2}\right)\right) \mathrm{m}, & \text { if } x<0 \mathrm{~m}, \\ 0.6 \exp \left(-100\left((x-0.5)^{2}+(y-0.5)^{2}\right)\right) \mathrm{m}, & \text { otherwise, }\end{cases}
$$

on a computational domain $[-1,1] \mathrm{m} \times[-1,1] \mathrm{m}$. The initial conditions are defined by a constant water level of $3 \mathrm{~m}$, quiescent flow and a constant $\theta$ satisfying (1.3a). In particular, the initial conditions are given by:

$$
h(x, y, 0)=3-B(x, y) \mathrm{m}, \quad u(x, y, 0)=0 \mathrm{~m} / \mathrm{s}, \quad v(x, y, 0)=0 \mathrm{~m} / \mathrm{s}, \quad \theta(x, y, 0)=\frac{4}{3} .
$$

We compute the solution at $t=0.12$ s on a mesh with $200 \times 200$ cells, and present the $L^{1}$ error norm in Table 4. The presented results prove that the proposed method exactly preserves the steady state even for the two-dimensional case.

Table 4: $L^{1}$ errors for different precisions for the lake at rest steady state solutions in Section 3.2.1.

\begin{tabular}{||c|cccc||}
\hline Precision & $h$ & $h u$ & $h v$ & $h \theta$ \\
\hline Single & $2.14 \times 10^{-7}$ & $2.63 \times 10^{-7}$ & $1.36 \times 10^{-8}$ & $2.33 \times 10^{-7}$ \\
\hline Double & $3.65 \times 10^{-15}$ & $4.28 \times 10^{-15}$ & $7.14 \times 10^{-15}$ & $1.85 \times 10^{-15}$ \\
\hline
\end{tabular}

\subsubsection{The small perturbation of a steady solution}

To test the capability of the DG method to cope with a small amplitude perturbation of a 2D steady solution over a non-flat bottom, we consider a topography containing two Gaussian shaped humps

$$
B(x, y)= \begin{cases}0.5 \exp \left(-100\left((x+0.5)^{2}+(y+0.5)^{2}\right)\right) \mathrm{m}, & \text { if } x<0 \mathrm{~m}, \\ 0.6 \exp \left(-100\left((x-0.5)^{2}+(y-0.5)^{2}\right)\right) \mathrm{m}, & \text { otherwise, }\end{cases}
$$

on a domain $[-1,1] \mathrm{m} \times[-1,1] \mathrm{m}$. Then, we consider the following preliminary initial conditions:

$$
(h, u, v, \theta)(x, y, 0)= \begin{cases}\left((3-B(x, y)) \mathrm{m}, 0 \mathrm{~m} / \mathrm{s}, 0 \mathrm{~m} / \mathrm{s}, \frac{4}{3}\right), & \text { if } x^{2}+y^{2}<0.25 \mathrm{~m}^{2}, \\ ((2-B(x, y)) \mathrm{m}, 0 \mathrm{~m} / \mathrm{s}, 0 \mathrm{~m} / \mathrm{s}, 3), & \text { otherwise, }\end{cases}
$$

which represent a steady solution for the system (2.14). Then, we perform a simulation until $t=0.12$ s on a mesh with $200 \times 200$ cells, and present the results in Fig. 4 . Looking at the figure we can conclude that the model is capable of preserving the steady state with negligible numerical diffusion without spurious oscillations. 

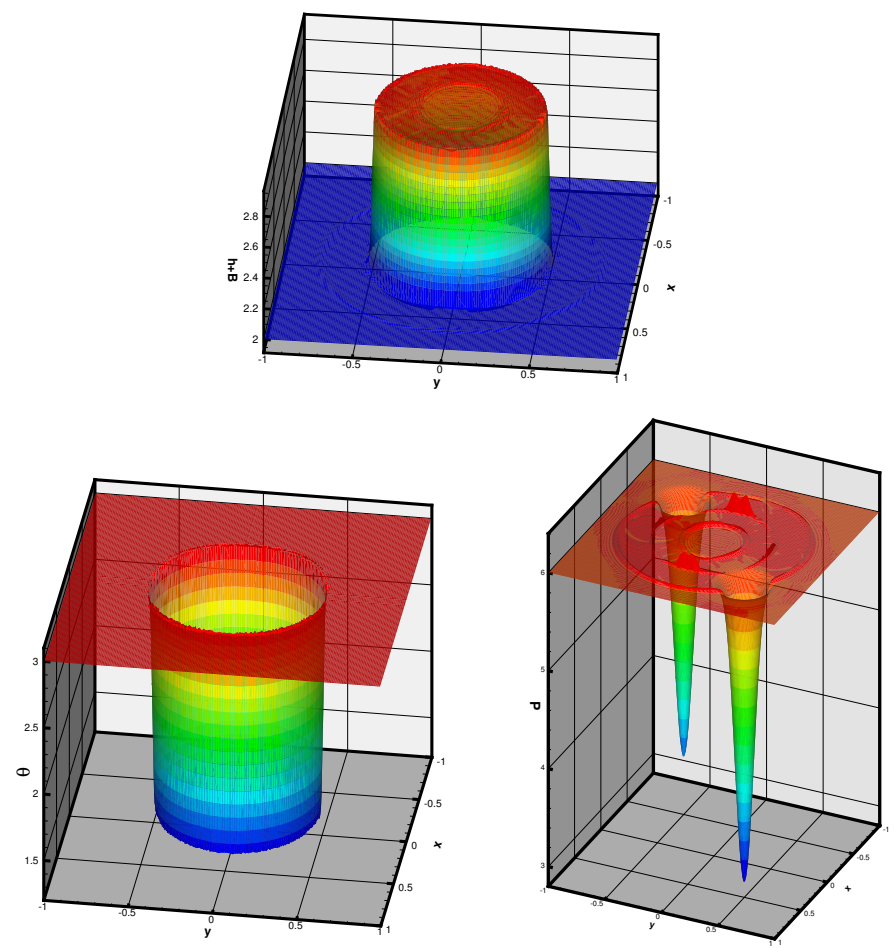

Figure 4: The numerical results of the steady state, Section 3.2.2, at time $t=0.12 \mathrm{~s}$ with $200 \times 200$ cells. $h+B$ (upper), $\theta$ (lower left) and $P=\frac{1}{2} g \theta h^{2}$ (lower right).

Successively we perturb the initial data as follows:

$$
= \begin{cases}\left(3-B(x, y)+0.1 \mathrm{~m}, 0 \mathrm{~m} / \mathrm{s}, 0 \mathrm{~m} / \mathrm{s}, \frac{4}{3}\right), & \text { if } 0.01 \mathrm{~m}^{2}<x^{2}+y^{2}<0.09 \mathrm{~m}^{2}, \\ \left(3-B(x, y) \mathrm{m}, 0 \mathrm{~m} / \mathrm{s}, 0 \mathrm{~m} / \mathrm{s}, \frac{4}{3}\right), & \text { if } 0.09 \mathrm{~m}^{2}<x^{2}+y^{2}<0.25 \mathrm{~m}^{2} \text { or } x^{2}+y^{2}<0.01 \mathrm{~m}^{2}, \\ (2-B(x, y) \mathrm{m}, 0 \mathrm{~m} / \mathrm{s}, 0 \mathrm{~m} / \mathrm{s}, 3), & \text { otherwise. }\end{cases}
$$

In Fig. 5 , we present the results at $t=0.15$ s on a mesh with $200 \times 200$ cells. We can clearly observe that, due to the well-balancing property, the method can reproduce the propagation of small perturbations of the steady state.

\subsubsection{The radial dam break over a flat bottom}

In the end, we consider a radial dam break problem over a flat bottom topography. The initial conditions are defined as follows:

$$
(h, u, v, \theta)(x, y, 0)= \begin{cases}(2 \mathrm{~m}, 0 \mathrm{~m} / \mathrm{s}, 0 \mathrm{~m} / \mathrm{s}, 1), & \text { if } x^{2}+y^{2}<0.25 \mathrm{~m}^{2} \\ (1 \mathrm{~m}, 0 \mathrm{~m} / \mathrm{s}, 0 \mathrm{~m} / \mathrm{s}, 1.5), & \text { otherwise }\end{cases}
$$



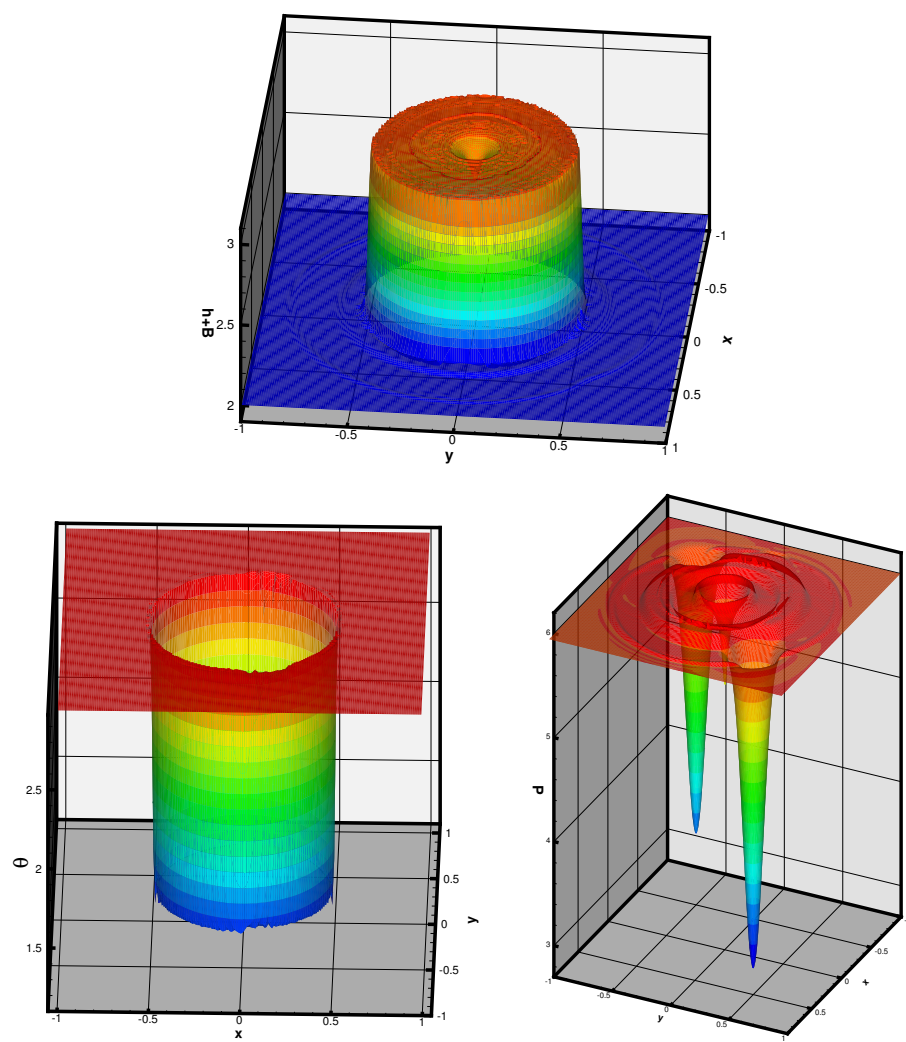

Figure 5: The numerical results of the small perturbation of a steady state solution in Section 3.2.2 at time $t=0.15$ s with $200 \times 200$ cells. $h+B$ (upper), $\theta$ (lower left) and $P=\frac{1}{2} g \theta h^{2}$ (lower right).

on a computational domain $[-1,1] \mathrm{m} \times[-1,1] \mathrm{m}$. We show the results at $t=0.15 \mathrm{~s}$ with $200 \times 200$ cells in Fig. 6 . We can clearly observe that the flow rapidly becomes transcritical due to the large difference in water depth and temperature inside and outside of the dam. In addition, we also present the scatter plots of the cross sections of the water height along the diagonal line $(y=x)$ and $y$-axis $(x=0)$, obtained on $50 \times 50,100 \times 100$, and $200 \times 200$ cells, respectively, in Fig. 7 and Fig. 8 . We obviously observe that the numerical results have good convergence.

\section{Concluding remarks}

In this research, we present a high-order DG method for the Ripa model. Well-balanced numerical fluxes with the help of the hydrostatic reconstruction idea as well as a direct source term approximation allow us to realize a well-balanced DG method, which maintains the lake at rest steady state at the discrete level. Both rigorous theoretical analysis and numerical examples are applied to validate the well-balancing property of the cur- 

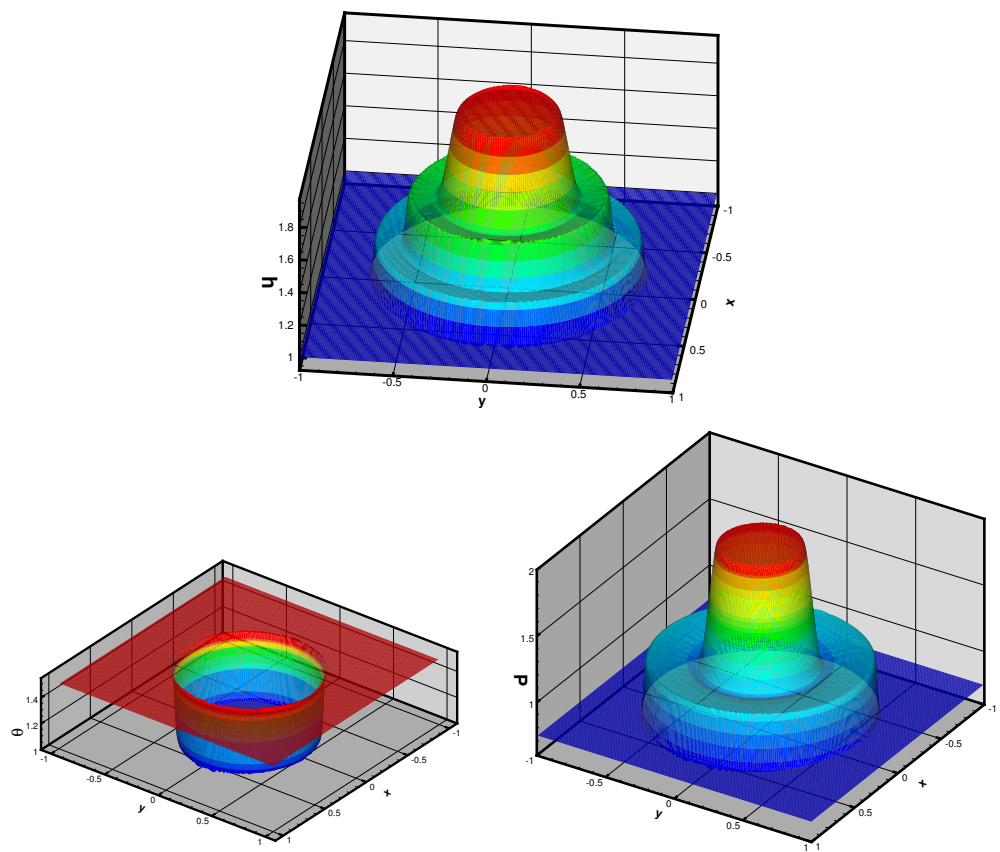

Figure 6: The numerical results of the radial dam break over the flat bottom, Section 3.2.3, at time $t=0.15 \mathrm{~s}$ with $200 \times 200$ cells. $h$ (upper), $\theta$ (lower left) and $P=\frac{1}{2} g \theta h^{2}$ (lower right).

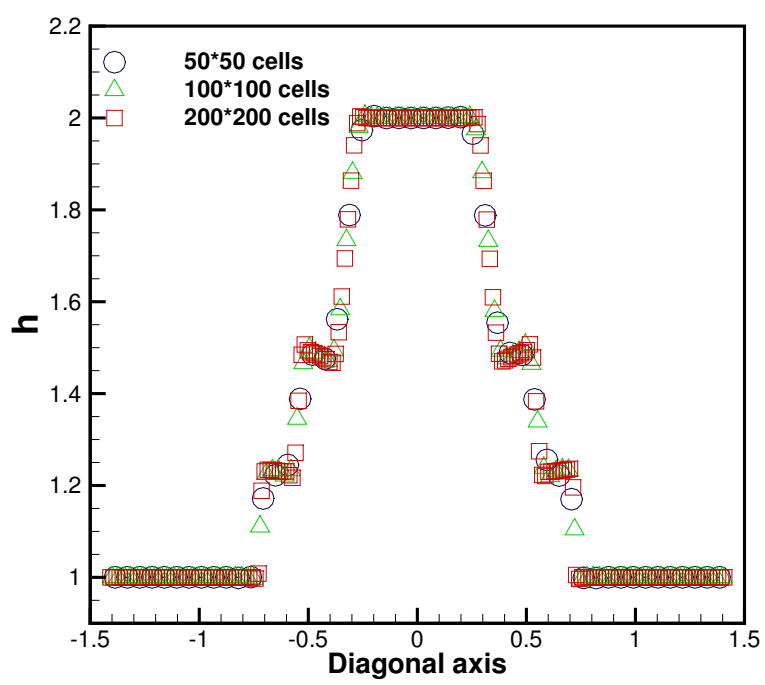

Figure 7: Scatter plot of the cross sections of the water depth $h$ at time $t=0.15$ s along the diagonal line $y=x$ obtained on $50 \times 50,100 \times 100$, and $200 \times 200$ cells in Section 3.2.3. 


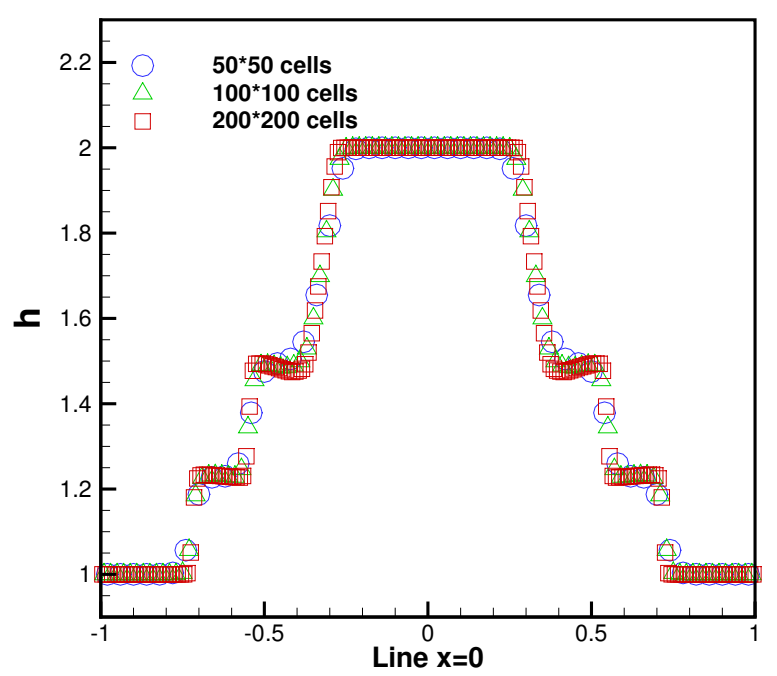

Figure 8: Scatter plot of the cross sections of the water depth $h$ at time $t=0.15$ s along the $y$-axis $(x=0)$ obtained on $50 \times 50,100 \times 100$, and $200 \times 200$ cells in Section 3.2.3.

rent DG method. Moreover, extensive results strongly suggest that the proposed method enjoys high-order accuracy for smooth solutions, and keeps good resolutions for discontinuous solutions at the same time.

\section{Appendix}

\section{A The eigenvalues of one-dimensional Ripa model}

The Jacobian matrix $\frac{\partial F(U)}{\partial U}$ has the following form:

$$
\frac{\partial F(U)}{\partial U}=\left(\begin{array}{ccc}
0 & 1 & 0 \\
\frac{1}{2} c^{2} \theta-u^{2} & 2 u & \frac{1}{2} c^{2} \\
-u \theta & \theta & u
\end{array}\right)
$$

with $c=\sqrt{g h}$ being the celerity. The eigenvalues are as follows:

$$
\lambda_{1}=u-c \sqrt{\theta}, \quad \lambda_{2}=u, \quad \lambda_{3}=u+c \sqrt{\theta} .
$$




\section{B The eigenvalues of two-dimensional Ripa model}

The Jacobian matrix in the $x$-direction has the following form:

$$
\frac{\partial F(U)}{\partial U}=\left(\begin{array}{cccc}
0 & 1 & 0 & 0 \\
\frac{1}{2} c^{2} \theta-u^{2} & 2 u & 0 & \frac{1}{2} c^{2} \\
-u v & v & u & 0 \\
-u \theta & \theta & 0 & u
\end{array}\right)
$$

with the following eigenvalues:

$$
\lambda_{1}=u-c \sqrt{\theta}, \quad \lambda_{2}=u, \quad \lambda_{3}=u, \quad \lambda_{4}=u+c \sqrt{\theta} .
$$

Similarly, the Jacobian matrix in the $y$-direction enjoys the below form:

$$
\frac{\partial G(U)}{\partial U}=\left(\begin{array}{cccc}
0 & 0 & 1 & 0 \\
-u v & v & u & 0 \\
\frac{1}{2} c^{2} \theta-v^{2} & 0 & 2 v & \frac{1}{2} c^{2} \\
-v \theta & 0 & \theta & v
\end{array}\right),
$$

which possesses the following eigenvalues in the form:

$$
\lambda_{1}=v-c \sqrt{\theta}, \quad \lambda_{2}=v, \quad \lambda_{3}=v, \quad \lambda_{4}=v+c \sqrt{\theta} .
$$

\section{Acknowledgements}

The second author Gang Li acknowledge the support of the Natural Science Foundation of China through Grants No. 11771228. The author Qiang Niu is supported by the XJTLU research enhancement fund with No. REF-18-01-04 and the Key Programme Special Fund (KSF) in XJTLU with Nos. KSF-E-32, KSF-E-21 and KSF-P-02. We sincerely thank Prof. Valerio Caleffi for his constructive guidance and revision of this manuscript.

\section{References}

[1] S. F. BRADFORD AND B.F. SANDERS, Finite volume model for shallow water flooding of arbitrary topography, J. Hydraulic Eng., 128 (2002), pp. 289-298.

[2] G. GotTARDi AND M. VenUtelli, Central scheme for the two-dimensional dam-break flow simulation, Adv. Water Res., 27 (2004), pp. 259-268.

[3] P. DELLAR, Common hamiltonian structure of the shallow water equations with horizontal temperature gradients and magnetic fields, Phys. Fluids, 303 (2003), pp. 292-297. 
[4] P. RIPA, Conservation laws for primitive equations models with inhomogeneous layers, Geophys. Astrophys. Fluid Dyn., 70 (1993), pp. 85-111.

[5] P. RIPA, On improving a one-layer ocean model with thermodynamics, J. Fluid Mech., 303 (1995), pp. 169-201.

[6] V. Desveaux, M. Zenk, C. Berthon and C. Klingenberg, Well-balanced schemes to capture non-explicit steady states: Ripa model, Math. Comput., 85 (2016), pp. 1571-1602.

[7] S. Noelle, Y. L. XING AND C.-W. SHU, High-Order Well-Balanced Schemes, Numerical Methods for Balance Laws, G. Puppo and G. Russo, editors, Quaderni di Mathematica, 2010.

[8] Y. L. XING, C.-W. SHU AND S. NOELLE, On the advantage of well-balanced schemes for movingwater equilibria of the shallow water equations, J. Sci. Comput., 48 (2011), pp. 339-349.

[9] X. F. WANG, G. LI, S. G. QIAN, J. J. Li AND Z. WANG, High order well-balanced finite difference WENO schemes for shallow water flows along channels with irregular geometry, Appl. Math. Comput., 363 (2019), 124587.

[10] X. WeN, Z. GAO, W. S. DON, Y. XING AND P. LI, Application of positivity-preserving wellbalanced discontinuous Galerkin method in computational hydrology, Comput. Fluids, 139 (2016), pp. 112-119.

[11] X.F. WANG, H. Y. YU, G. LI AND J. M. GAO, Hybrid finite volume weighted essentially nonoscillatory schemes with linear central reconstructions, Appl. Math. Comput., 359 (2019), pp. 132-147.

[12] J. M. GREenberG AND A.-Y. LeRoux, A well-balanced scheme for the numerical processing of source terms in hyperbolic equations, SIAM J. Numer. Anal., 33 (1996), pp. 1-16.

[13] J. M. GreenberG, A.-Y. LeRoux, R. BAraille And A. Noussair, Analysis and approximation of conservation laws with source terms, SIAM J. Numer. Anal., 34 (1997), pp. 1980-2007.

[14] A. BERMÚdeZ AND M. E. VÁZQUEZ, Upwind methods for hyperbolic conservation laws with source terms, Comput. Fluids, 23 (1994), pp. 1049-1071.

[15] A. Bermúdez, A. Dervieux, J.-A. Desideri And M. E. VÁzquez, Upwind schemes for the two-dimensional shallow water equations with variable depth using unstructured meshes, Comput. Methods Appl. Mech. Eng., 155 (1998), pp. 49-72.

[16] A. CHertock, A. KuRGanOv AND Y. LiU, Central-upwind schemes for the system of shallow water equations with horizontal temperature gradients, Numerische Mathematik, 127 (2014), pp. 595-639.

[17] A. CHERTOCK, S. KARNI AND A. KuRGANOV, Interface tracking method for compressible multifluids, ESAIM: Mathematical Modelling and Numerical Analysis, 42 (2008), pp. 991-1019.

[18] R. Touma AND C. KLingenberG, Well-balanced central finite volume methods for the Ripa system, Appl. Numer. Math., 97 (2015), pp. 42-68.

[19] C. Sánchez-Linares, T. Morales de lunab and M. J. Castro Díaz, A HLlC scheme for Ripa model, Appl. Math. Comput., 272(2) (2016), pp. 369-384.

[20] X. HAN AND G. LI, Well-balanced finite difference WENO schemes for the Ripa model, Comput. Fluids, 134-135 (2016), pp. 1-10.

[21] S. G. QIAN, F. J. SHAO AND G. LI, High order well-balanced discontinuous Galerkin methods for shallow water flow under temperature fields, Comput. Appl. Math., 37 (2018), pp. 5775-5794.

[22] B. COCKBURN, G. KARNIADAKIS AND C.-W. SHU, The development of discontinuous Galerkin methods, in: B. Cockburn, G. Karniadakis, C.-W. Shu (ed.), Discontinuous Galerkin Methods: Theory, Computation and Applications, Lecture Notes in Computational Science and Engineering, Part I: Overview, 3-50, Springer, 2000.

[23] C.-W. SHU, High order WENO and DG methods for time-dependent convection-dominated PDEs: A brief survey of several recent developments, J. Comput. Phys., 316 (2016), pp. 598-613. 
[24] F. Aureli, A. Maranzoni, P. MignosA And C. A. Ziveri, Weighted surface-depth gradient method for the numerical integration of the 2D shallow water equations with topography, Adv. Water Res., 31 (2008), pp. 962-974.

[25] A. ERN, S. PIPERNO AND K. DJADEL, A well-balanced Runge-Kutta discontinuous Galerkin method for the shallow-water equations with flooding and drying, Int. J. Numer. Methods Fluids, 58 (2008), pp. 1-25.

[26] A. CAnestrelli, A. Siviglia, M. Dumbser And E. F. TORO, Well-balanced high-order centred schemes for non-conservative hyperbolic systems, Applications to shallow water equations with fixed and mobile bed, Adv. Water Res., 32 (2009), pp. 834-844.

[27] G. Kesserwani, Q. LiAnG, J. VAzQueZ And R. Mosé, Well-balancing issues related to the RKDG scheme for the shallow water equations, Int. J. Numer. Methods Fluids, 62 (2010), pp. 428-448.

[28] F. Benkhaldoun, I. Elmahi And M. SEAÏD, A new finite volume method for flux-gradient and source-term balancing in shallow water equations, Comput. Methods Appl. Mech. Eng., 199 (2010), pp. 3224-3335.

[29] G. KESSERWANI AND Q. H. LIANG, A discontinuous Galerkin algorithm for the two-dimensional shallow water equations, Comput. Methods Appl. Mech. Eng., 199 (2010), pp. 3356-3368.

[30] S. OSTKAMP, Multidimensional characteristic Galerkin methods for hyperbolic systems, Math. Methods Appl. Sci., 20(13) (1997), pp. 1111-1125.

[31] J. KOU AND S. SUN, Analysis of a combined mixed finite element and discontinuous Galerkin method for incompressible two-phase flow in porous media, Math. Methods Appl. Sci., 37(7) (2013), pp. 962-982.

[32] G. LI, L. N. SONG AND J. M. GAO, High order well-balanced discontinuous Galerkin methods based on hydrostatic reconstruction for shallow water equations, J. Comput. Appl. Math., 340 (2018), pp. 546-560.

[33] E. Audusse, F. Bouchut, M.-O. Bristeau, R. Klein and B. Perthame, A fast and stable well-balanced scheme with hydrostatic reconstruction for shallow water flows, SIAM J. Sci. Comput., 25 (2004), pp. 2050-2065.

[34] C.-W. SHU, Total-variation-diminishing time discretizations, SIAM J. Sci. Statistical Comput., 9 (1988), pp. 1073-1084.

[35] C.-W. SHU, TVB uniformly high-order schemes for conservation laws, Math. Comput., 49 (1987), pp. $105-121$.

[36] B. COCKBURn AND C.-W. SHU, The Runge-Kutta discontinuous Galerkin method for conservation laws V: multidimensional systems, J. Comput. Phys., 141 (1998), pp. 199-224.

[37] A. REHMAN, I. Ali AND S. QAMAR, Exact Riemann solutions of the Ripa model for flat and non-flat bottom topographies, Results Phys., 8 (2018), pp. 104-113. 